\title{
Development of Driving Jack System for Tunnel Boring Machine
}

\author{
Fumihiko Ishise*,Keizo Kazama**,Shiro Honmura***, \\ Makoto Sameshima***
}

* Kobe Shipyard \& Machinery Works, Mitsubishi Heavy Industries, Ltd., 1-1-1 Wadasaki-cho, Hyogo-ku, Kobe 652, JAPAN

** Civil Engineering Technical Division, Obayashi Corp., 2-2-9 Hongo, Bunkyo-ku, Tokyo 113, JAPAN

*** Takasago Research \& Development Center, Mitsubishi Heavy Industries, Ltd., 2-1-1 Niihama, arai-cho, Takasago 676, JAPAN

\begin{abstract}
To realize stable excavation control on unsound ground and linear control along with scheduled lines when excavation operations are performed with tunnel boring machines,etc., driving jack systems have been developed as an application of parallel links which enable us to use position/posture controls with 6 degrees of freedom. This tunnel boring machine is subjected to combined great loads such as traveling, rotational torque, radial force,etc. during excavation operations. The method of mechanical analysis and the control approach have been finalized with redundant axes. Bench tests found the performance to be appropriate. As a result, the prospects of its application in production machines have been confirmed by ensuring that the load capacity of and load distribution of driving jack systems will almost agree with calculated values, and that control accuracy and position/posture holding capacities will have no problem.
\end{abstract}

\section{INTRODUCTION}

An attempt has been made to improve the work environment and to pursue the safety and economical efficiency at the building site in these years, and the positive effort has been made correspondingly to develop or introduce the building robot, automation machine, etc. The similar problems have become severer even in the field of tunnel execution. Consequently, the increased demand has been given on 
the improvement of execution efficiency on the unsound ground, correspondence to quick-curve traveling, etc., even for driving jack system for tunnel boring machine (hereafter to be called "TBM" in short).

The TBM is a machine which is so designed as to support the body by the gripper arranged at the rear shell, and to bore the rock bed by pushing the roller cutter (arranged at the front surface of frontshell) against the rock bed, while turning it. It is necessary for the driving jack system to protrude the front shell along the scheduled line of tunnel, while supporting the excavation reaction force. Consequently, the driving jack system for TBM, using the parallel link was developed, for improvement of execution efficiency/direction control accuracy, and automation of direction control.

Since the actuator is arranged in parallel in the system using parallel link, the system is featured by that it is possible for the parallel link to generate a great force and increase rigidity, and that it provides a limited operation range. ${ }^{1 \text { ) }}$ To ensure the desired load distribution against big load generated during excavation, the paper describes the analysis method and control method of position/posture for the parallel link mechanism that the parallel link system of more than 7 axes is configured. ${ }^{2)}$

\section{Parallel link mechanism}

Fig.1 shows the concept configuration drawing of parallel link driving jack system. To control 6 degrees of freedom for end plate position/posture, the necessary/sufficient condition for driving link is "6 axes". But, the driving link was designed for 10 axis link configuration (redundant parallel link mechanism), taking big load generated during boring into account. The investigation was executed for the kinematics and statics shown below, which are required for driving force calculation and position/posture control.

\subsection{Kinematics}

If the base joint coordinate value is substituted for "Bi", and that of end joint for "Ei", the link vector "Ui" is given by the equation-(1).

$$
\mathbf{U i}=\mathbf{E} \mathrm{i}-\mathbf{B i}
$$

Consequently, the link length "Li" is given as follows;

$$
\mathbf{L i}=|\mathbf{U i}|
$$

Since it is normally unable to find the forward kinematics analytically, on the other hand, it found by repetitive approximation calculation. The following relationship is established for slight displacement between the link length $\mathbf{L}$ and end plate position posture $\mathbf{X}$.

$$
\delta \mathbf{L}=\mathbf{J} \delta \mathbf{X}
$$

where, J: Jacobian 
The end plate and base plate are thought to be a rigid body, and the position/ posture of end plate can be found from 6 any links. The followings are given from the equation- (3).

$$
\begin{aligned}
& \delta \mathbf{X}^{*}=\mathbf{J}^{*-1} \delta \mathbf{L}^{*} \\
& \mathbf{X}(\mathrm{n})=\mathbf{X}(\mathrm{n}-1)+\mathbf{J}^{*-1} \delta \mathbf{L}^{*}(\mathrm{n}-1)
\end{aligned}
$$

where, ${ }^{*}: \delta \mathbf{X}, \delta \mathbf{L}, \mathbf{J}$ at any 6 links

The repetitive calculation was made, using the equations (4) and (5) until $\left|\delta \mathbf{L}^{*} \mathbf{i}\right|$ $<\varepsilon$ could be assured.

\subsection{Statics}

If the force vector is substituted for "F", and link driving force for "C", the following is given;

$$
\mathbf{F}^{\mathrm{T}} \delta \mathbf{X}=\mathbf{C}^{\mathrm{T}} \delta \mathbf{L}
$$

The forward statics and inverse statics can be given from the equations (3) and (6) as follows;

$$
\begin{aligned}
& \mathbf{F}=\mathbf{J}^{\mathrm{T}} \mathbf{C} \ldots . . \\
& \mathbf{C}=\left(\mathbf{J}^{\mathrm{T}}\right){ }^{\prime} \mathbf{F}
\end{aligned}
$$

For the mechanism having link of more than 7 axes, it is unable to determine the combination of link driving force univocaly, which satisfies the generation force of the end plate. The equation-(8) is used to solve the minimum square sum value for link driving force, and $\left(\mathbf{J}^{\mathrm{T}}\right)^{\prime}$ indicates a pseudo inverse transposed Jacobian matrix.

Fig.2 shows an example of calculation result of 6 axis, 10 axis system, in which the result of calculation executed, using the DADS ( Dynamic Analysis Design System ), one of mechanism analysis programs, is indicated side by side. The results of both calculation methods are well consistent, and it is thought possible to examine the general particulars of link, to execute the dynamic analysis by utilizing the calculation method or mechanism analysis program. It is also known that the load is distributed in case of 10 axis system, as the link driving force in this case is smaller as compared with that of 6 axis system.

\section{Testing apparatus}

\subsection{Configuration of testing apparatus}

The testing apparatus was assumed to be a $1 / 2$ scale model of actual equipment $(\phi 3.5 \mathrm{~m})$, of which assembly drawing is shown in Fig.3, and general particulars in Table -1 respectively. 
Table-1 Main particulars of testing apparatus

\begin{tabular}{|l|c|c|c|}
\hline & $\begin{array}{c}\text { Testing } \\
\text { apparatus }\end{array}$ & $\begin{array}{c}\text { Actual } \\
\text { equipment }\end{array}$ \\
\hline \multicolumn{2}{|l|}{ Parallel link P.C.D. } & $\phi 1,390 \mathrm{~mm}$ & $\phi 3,500 \mathrm{~mm}$ \\
\hline Parallel link stroke & $600 \mathrm{~mm}$ & $1,200 \mathrm{~mm}$ \\
\hline \multirow{2}{*}{$\begin{array}{l}\text { Load } \\
\text { capacity }\end{array}$} & Thrust & $2,180 \mathrm{kN}$ & $8,700 \mathrm{kN}$ \\
\cline { 2 - 4 } & Radial & $50 \mathrm{kN}$ & - \\
\cline { 2 - 4 } & Torque & $125 \mathrm{kN} \cdot \mathrm{m}$ & $1,000 \mathrm{kN} \cdot \mathrm{m}$ \\
\hline \multicolumn{2}{|l|}{ Link cylinder size } & $\phi 125 \mathrm{~mm} / \phi 71$ & - \\
\hline
\end{tabular}

The base plate and end plate were connected to both ends of each cylinder of parallel link, using the spherical joint. And each cylinder was arranged with magnetostriction type displacement meter and pressure gauge. Two thrust load cylinders and radial load cylinder were arranged at right and left of end plate, which made it possible to apply three different kinds of thrust, radial or torque loads independently or compositely. The roller mechanism was also arranged at the under side of radial load cylinder, to allow the movement in the thrust direction, while applying the radial and torque loads.

\subsection{Control unit}

The servo valve was used to drive the cylinder. The displacement command value sent from the personal computer, and feedback signal sent from the cylinder displacement meter were used as input signal for servo valve. The target value for end plate was input from the joystick in case of manual operation, and from the personal computer in case of automatic operation. In performing the test, the parallel link cylinder was controlled for displacement, while controlling the load cylinder.

\subsection{Control program}

The outline configuration of control program is as shown in Fig.4. The control sampling time for automatic operation is $20 \mathrm{~ms}$. The time for repetitive approximation calculationin forward kinematics per one loop is $1 \mathrm{~ms}$. When the initial value is unknown, it is necessary to execute the repetitive calculation $4 \sim 5$ times. While the realtime control was executing, however, the operation converged by the repetitive calculation of approx. $1 \sim 2$ times. The repetitive approximation calculation in forward kinematics is said to be prospective to secure the sufficient realtime performance. 


\section{Test items and results}

The actual measurement length and command length of cylinder in operation, cylinder driving force and load cylinder pressure were recorded in the hard disc built in the control unit. After completion of test, the load characteristics and position/posture were subjected to calculation processing using the data processing program.

\subsection{Load capacity test}

To confirm the end plate for load support capacity, the thrust load, radial load and torque load were applied independently or compositely, while keeping the end plate at the fixed position/posture, to measure the end plate for displacement, and each cylinder for load distribution.

Fig. 5 shows the actual measurement driving force distributed to the cylinder and calculated driving force for case when the thrust load, radial load and torque load were applied compositely, in which the actual measurement value was almost consistent with the calculated value, proving that the analysis method used in the chapter- 2 is thought to be almost proper.

\subsection{Step load test}

To confirm the position/posture holding performance for case when the dynamic load is applied, the load was changed stepwise, while holding the end plate at the fixed position/posture, to measure the end plate for displacement, and the cylinder for driving force respectively.

Fig.6 shows the actual measurement value of load force for case when the thrust force is applied at $500 \mathrm{~mm}$ stroke position as well as the force generated at the end plate, calculated from the cylinder driving force, in which the force generated well follows the load force. The change in posture in this case is small, and can be said to provide the position/posture holding performance as designed.

\subsection{Straight traveling/curved travelling test}

Taking the practical excavation condition into consideration, the end plate was advanced at a speed of $50 \mathrm{~mm} / \mathrm{min}$, while applying constant load to the end plate, to measure the end plate for position/posture, and the cylinder for driving force. The test was performed at the stroke of $500 \mathrm{~mm}$ for two cases of straight traveling and curved traveling. For curved traveling, the end plate traveled the center orbit of straight line given by connecting the start point to the end point of arc along R $15 \mathrm{~m}$, while changing the posture so that the end plate surface is directed toward the center of radius of curvature. 
Fig.7(a) shows the change in position/posture for case when the end plate is traveled curvedly in rightward direction, while applying the composite load. The control accuracy is $2 \sim 3 \mathrm{~mm}$. It was possible to confirm that the it was controlled to the tolerance of $\pm 5 \mathrm{~mm}$ or less within the entire stroke. It is known that the end plate is turning, while being displaced approx. $7 \mathrm{~mm}$ in right direction and approx. $1.9^{\circ}$ in angle, which indicates that the end plate travels curvedly in accordance with the command value.

The change in cylinder driving force in this case is shown in Fig.7(b), in which the driving force of each cylinder was found not to be excessive, but to be changing smoothly, and thus it was known that each cylinder was advancing, while supporting the load in harmonization.

\section{Consideration}

The following shows the items proved through the analysis and experiment described above.

\section{1) Mechanical analysis method}

The load capacity and load distribution of driving jack system are consistent with the calculated values, thus providing the sufficient rigidity. This indicates that the analysis method for parallel link including redundant axis is thought to have been able to be confirmed for validity. The investigation on this paper was made on the 10 axis system, as an example of parallel link system including the redundant axis of 7 axes or over. The method used in this case can be also used for parallel link system other than 10 axis. Consequently, it is possible to select the proper number of axes, depending upon the specifications of TBM, and thus thought possible to design the driving jack system corresponding to the specifications of practical equipment.

2) Control method

The displacement behavior of end plate and control accuracy during straight traveling/curved traveling during the stepwise load application of this test were found to be within the target value. This indicates that the control method makes it possible to control the position of parallel link system including redundant axis.

3) Grasping the external force

Since it is possible to calculate 6 components of load acting on the end plate, from the cylinder driving force in the driving jack system of parallel link, it becomes possible to find the load of main bearing to support the cutter head all the time, allowing to control the life of bearing. Besides, it is thought possible to utilize the method to gain the data or grasping of soil condition during boring. 
4) Direction control system

As compared with the conventional direction control method by force control, it is possible to further expect the improvement of execution control for TBM, such as improvement of linear control function in accordance with the scheduled line, etc. to directly control the position of cutter head. Since it is difficult for the operator to grasp the driving direction of end plate from the change in cylinder displacement in case of the parallel link, it is indispensable to execute the automatic direction control. It is thought that the automatic direction control can be realized through the linkage of control system and measurement data.

\section{Conclusion}

The development of driving jack system of TBM to which parallel link is applied could provide the following achievements.

1) It is able to establish the method for mechanical analysis and posture/position control of 10 axis parallel link system including redundant axis.

2) The bench test result made it possible to forecast the application to practical equipment after confirming that there is no problem in the load capacity and control accuracy of driving jack system.

3) The method used for this study is thought to be extendable to the system of other than 10 axis.

The authors are intended to utilize the function of this system effectively to further develop the direction control system including the gripper, and execution control system, etc..

\section{Reference}

1) T. Arai, et al. ; Design, Analysis and Construction of a Prototype Parallel Link Manipulator, Proc. IEEE Intl. Workshop on Intelligent Robots and Systems, pp.205-212, 1990

2) S. Tadokoro, Control of Parallel Mechanisms, JRSJ, Vol.10, No.6, 1992 

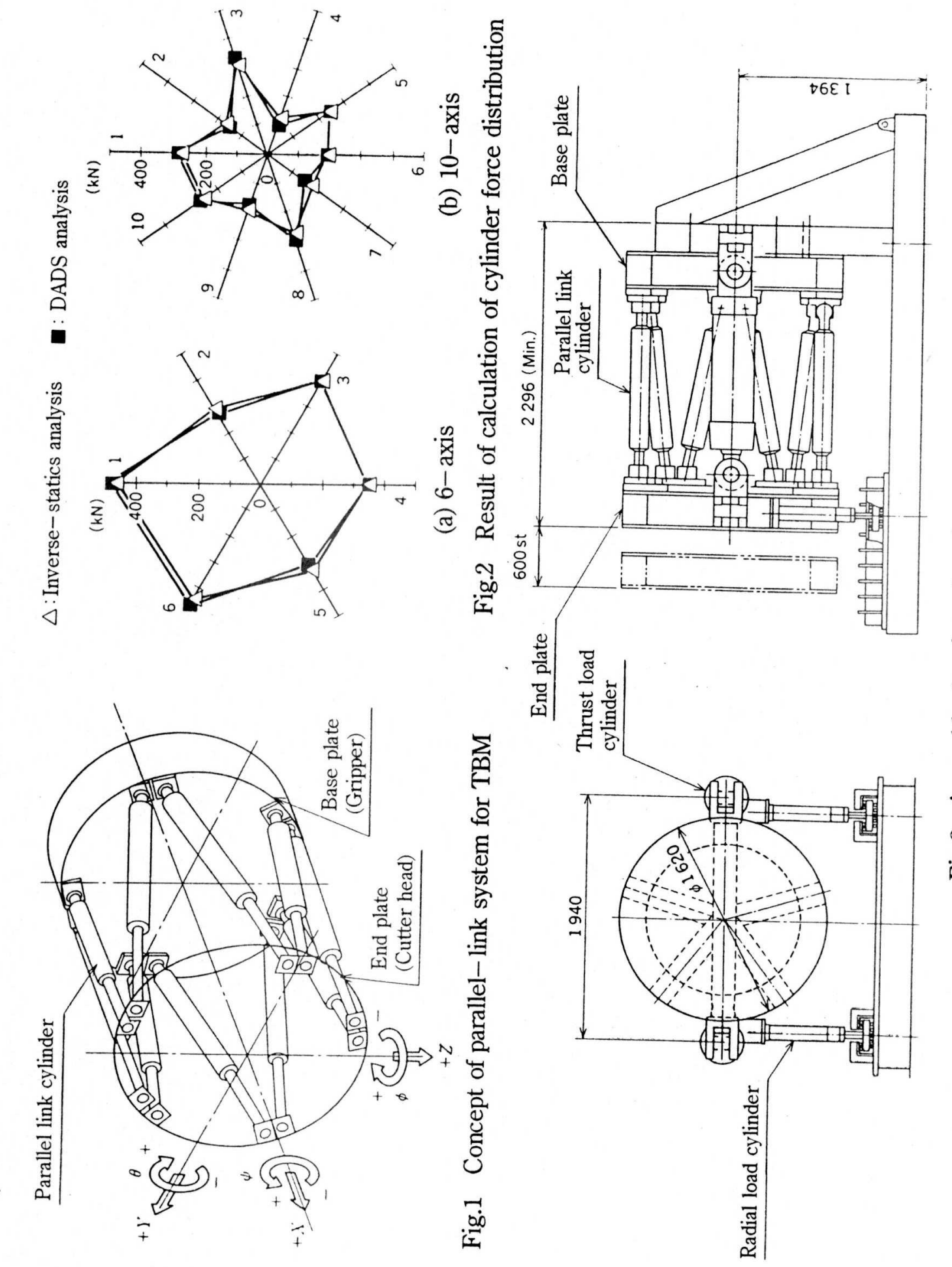

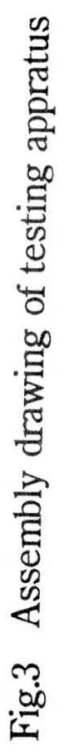



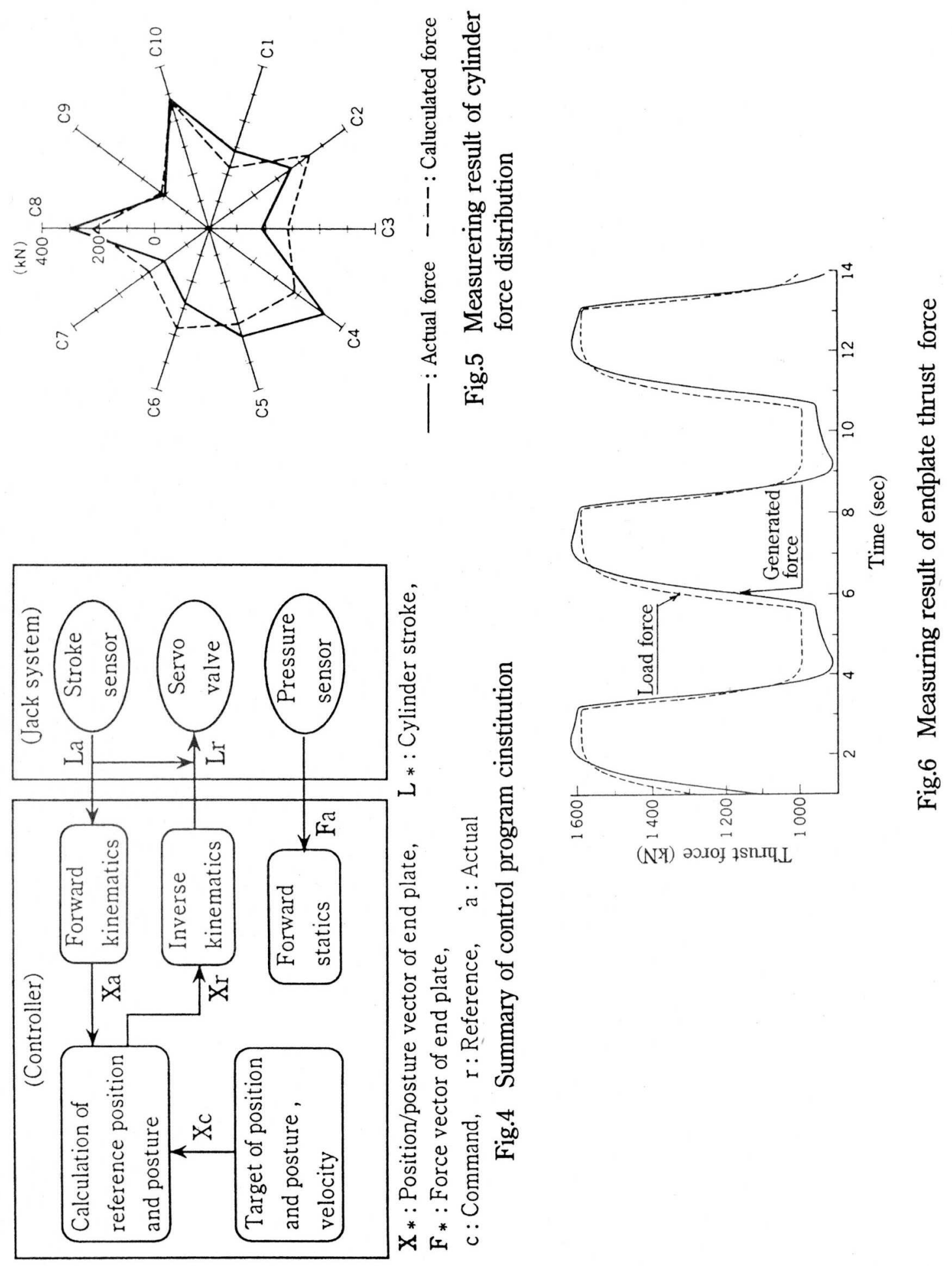

(NY) วगxof 7sn.पL 

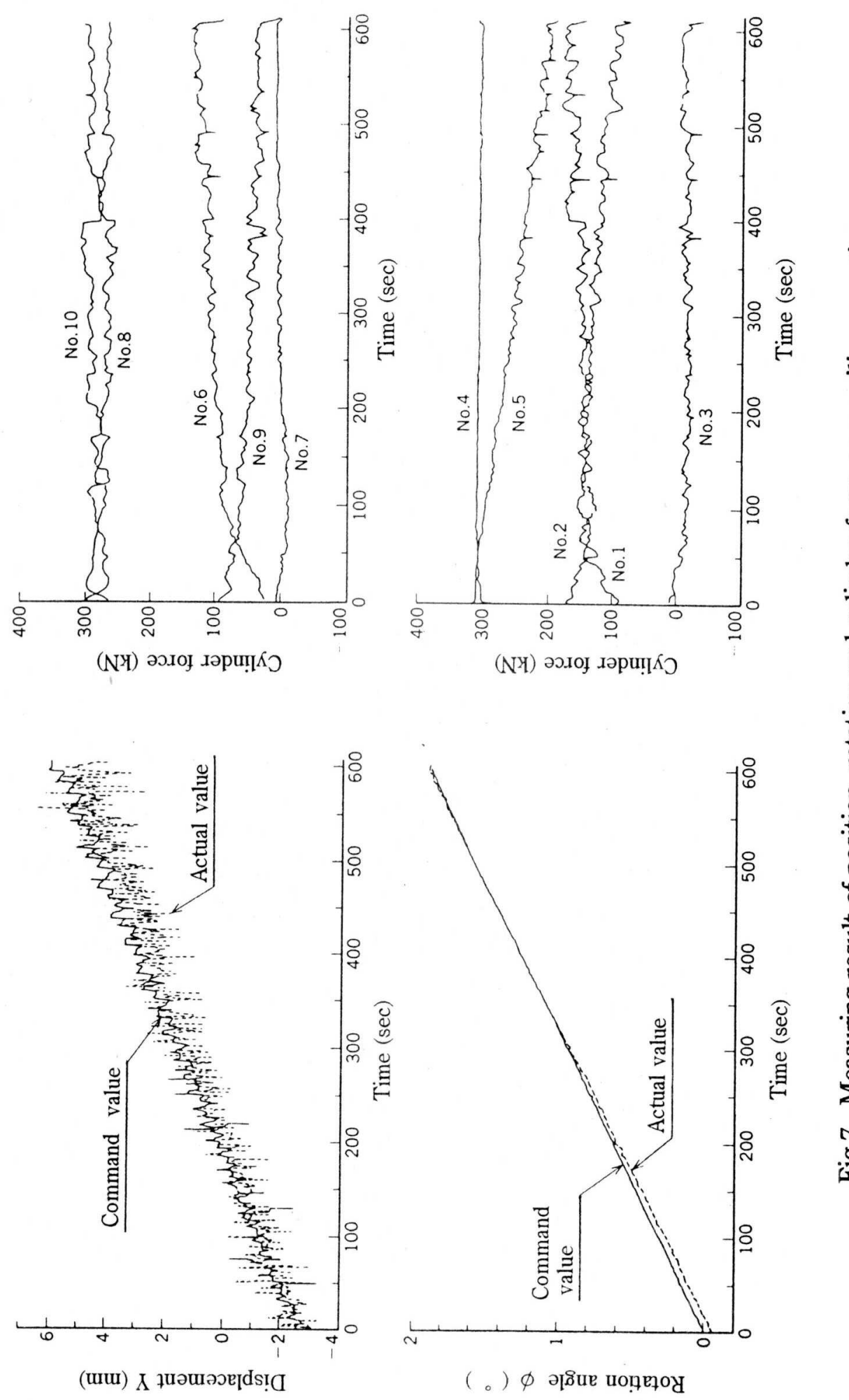

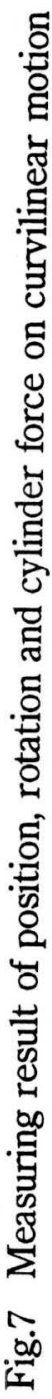

